\title{
Espacios de diálogo para la transición hacia el modelo social de atención a la personas con discapacidad: Una mirada desde la investigación acción participativa
}

\author{
Dialogue Spaces for the Transition to the Social Model of Care for People With Disabilities: A \\ Look From the Participatory Action Research
}

\section{Espaços de diálogo para a transição ao modelo social de atenção a pessoas com deficiência: uma perspectiva desde a investigação ação participativa}

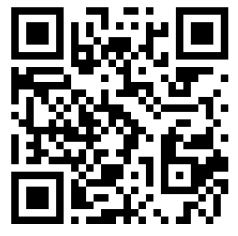

Eugenia Córdoba-Warner

Ministerio de Educación Pública

Centro de Educación Especial de Alajuela

Alajuela, Costa Rica

eucordoba@gmail.com

https://orcid.org/0000-0002-4592-1626

Recibido • Received • Recebido: 03 / 05 / 2018

Corregido • Revised • Revisado: 23 / 09 / 2019

Aceptado $\cdot$ Accepted $\cdot$ Aprovado: $13 / 11 / 2019$

\begin{abstract}
Resumen: El propósito del presente artículo es exponer la necesidad de una evolución del modelo rehabilitador al modelo social de atención a las personas con discapacidad en los centros de educación especial. Mediante la investigación acción participativa (IAP) se investigó la dinámica de un equipo profesional del servicio de estimulación temprana de discapacidad múltiple en un centro de educación especial conformado por una docente de educación especial, una terapeuta de lenguaje y dos fisioterapeutas. La investigación se llevó a cabo por medio de la sistematización de experiencias de seis encuentros del equipo interdisciplinar constituido en comunidad de aprendizaje. Las participantes descubrieron que tenían diferentes visiones acerca del mismo hecho y que pensando estar actuando desde un modelo social, continuaban actuando en muchas ocasiones con el modelo rehabilitador, como así lo demostró su posicionamiento ante el estudiantado y, sobre todo, ante las familias. Se concluye que se deben abrir espacios de diálogo donde los equipos interdisciplinarios puedan ser fuente de cambio en la comprensión de los procesos educativos y de la complejidad de las relaciones con los niños y las niñas y sus familias, desde un posicionamiento basado en los derechos humanos como es el modelo social de atención a las personas con discapacidad.
\end{abstract}

Palabras claves: Equipo interdisciplinario; transformación; sistematización; atención temprana; discapacidad. 
doi: http://doi.org/10.15359/ree.24-1.20

URL: http://www.una.ac.cr/educare

CORREO: educare@una.cr

\begin{abstract}
This article aims to expose the need for an evolution of the rehabilitation model to the social model of care for people with disabilities in special education centers. Through the participatory action research (PAR) approach, the dynamics of a professional team of the multiple disability early stimulation service at a special education center was studied; this center has a special education teacher, a language therapist, and two physical therapists. The research was conducted through the systematization of experiences of six meetings held by the interdisciplinary team, constituted as a learning community. The participants discovered that they had different visions about the same fact. They also found that thinking that they were acting under a social model, they continued to work, in many occasions, under the rehabilitation model, as demonstrated by their position towards students and, especially, to families. It is concluded that dialogue spaces must be opened where interdisciplinary teams can be a source of change in the understanding of educational processes and the complexity of relationships with children and their families; these spaces can be opened from a perspective based on human rights, as it is the social model of care for people with disabilities.
\end{abstract}

Keywords: Interdisciplinary team; transformation; systematization; family; early stimulation; disability.

Resumo: A finalidade do presente artigo éexpor a necessidade de uma evolução do modelo reabilitador ao modelo de assistência a pessoas com deficiência nos centros de educação especial. Através da investigação ação participativa (IAP) foi pesquisada a dinâmica de uma equipe profissional de serviço de estimulação precoce de deficiência múltipla num centro de educação especial conformado por uma docente de educação especial, uma terapeuta de linguagem e dois fisioterapeutas. A investigação aconteceu mediante a sistematização de experiências de seis encontros da equipe interdisciplinar constituída na comunidade de aprendizagem. As participantes descobriram que tinham diferentes visões sobre o mesmo fato e que pensavam estar atuando desde um modelo social, continuavam atuando em muitas ocasiões com o modelo reabilitador, como assim demonstra seu posicionamento frente aos estudantes e também as famílias. Conclui-se que devem ser abertos espaços de diálogo onde as equipes interdisciplinares possam ser partícipes na compreensão dos processos educativos e na complexidade das relações com as crianças e suas famílias, desde um posicionamento baseado nos direitos humanos como é o modelo atual de assistência social as pessoas com incapacidade.

Palavras chave: Equipe interdisciplinar; transformação;sistematização; atenção precoce;incapacidade.

\title{
Introducción
}

No existe separación entre aprender y vivir.

(Gallegos, 2001, p. 27)

La discapacidad es definida por la Organización Mundial de la Salud (OMS, 2011) "como un término genérico que engloba deficiencias, limitaciones de actividad y restricciones para la participación" (p. 7). La discapacidad es el resultado de la interacción de las personas con alguna condición de salud y los factores personales y ambientales. Es por esta razón que profesionales que trabajan con esta población se deben ocupar de estimulary apoyar las capacidades existentes en cada niño o niña y su familia, a la vez que realizan acciones de mejora y optimización de los factores ambientales para que la participación de las personas con discapacidad sea mayor.

2

Eugenia Córdoba-Warner

Los artículos de la Revista Electrónica Educare del Centro de Investigación y Docencia en Educación de la Universidad Nacional, Costa Rica, se comparten bajo términos de la Licencia Creative Commons: Reconocimiento, № Comercial, Sin Obra Derivada 3.0 Costa Rica. Las autorizaciones adicionales a las aquí delimitadas se pueden obtener en el correo: educare@una.cr 
Son multiples las condiciones de salud que pueden generar una discapacidad en la primera infancia (de cero a tres años) como: síndrome de down, prematuridad, bajo peso al nacer, retardo del desarrollo psicomotor, parálisis cerebral, entre otras. En Costa Rica, la población que presenta riesgo o alteraciones en el desarrollo es atendida mayoritariamente en los centros de educación especial. Concretamente, el servicio de estimulación temprana en discapacidad múltiple atiende personas que requieren:

- Sistemas alternativos para la comunicación.

- Adaptaciones y rehabilitación para el mejor desempeño físico y motor.

- Establecimiento del repertorio básico de comportamiento para el aprendizaje y para una adecuada interacción social.

- Apoyos intensos para el desempeño de actividades de la vida cotidianda, entre otros. (Ávila et al., 2005, p. 13)

La frase del inicio del apartado refleja un acercamiento a la investigación acción participativa (IAP en adelante por sus iniciales). En este tipo de investigación, la vida y el aprendizaje se mezclan y se funden en una sola experiencia enriquecida por el compartir cercano y abierto de quienes coinvestigan. En este artículo se quiso compartir la vivencia de cambio dentro de un equipo multidisciplinario y la evolución de este mismo hacia la interdisciplina por medio del diálogo que lleva a la reflexión, la acción y transformación en una espiral sin fin.

El proceso de investigación se realizó con el equipo de profesionales del servicio de estimulación temprana de discapacidad múltiple de un centro de educación especial. En la investigación participaron una docente de educación especial, una terapeuta del lenguaje y dos fisioterapeutas, una de ellas como investigadora principal. A inicios del 2016 este equipo tomó la decisión de realizar capacitaciones para las madres de familia con temáticas que fueran de su interés, especialmente enfocadas en tópicos relacionados con parálisis cerebral, que es la condición más común entre estudiantes. Los talleres se impartian una vez al mes, con una reunión previa dos semanas antes para planificarlo, estos espacios también brindaban la oportunidad de conversar sobre lo sucedido en el taller anterior. Al ir comprendiendo la discapacidad como el resultado de la interacción entre la condición de salud y los factores personales y ambientales, surgió la inquietud de analizar la práctica profesional con el fin de mejorar la atención que brinda a las familias de los niños y niñas.

La inquietud de la investigadora principal fue cuestionar si el equipo multidisciplinar tenía claro lo que deseaba hacer y si lo realizado hasta el momento respondía a los valores y principios de cada participante acerca de la atención de la persona con discapacidad y sus familias. El proceso permitió desarrollar estrategias de escucha y autoescucha; por medio del diálogo se aprendió a colocar una mirada sobre el hacer y a cuestionar por medio de la sistematización. 
doi: http://doi.org/10.15359/ree.24-1.20

URL: http://www.una.ac.cr/educare

CORREO: educare@una.cr

Para construir la espiral entre el accionar y el reflexionar se utilizó la estrategia de sistematización de la experiencia, con el objetivo de ordenar y reconstruir lo vivido y así analizar los factores que intervienen, cómo se relacionan entre sí y el porqué se ha dado de esta forma (Jara, 1994).

El proceso de sistematización efectuado condujo al equipo a analizar la propia práctica en relación al trabajo realizado con las familias, y cuestionar si este respondía a las necesidades de estas o a las del equipo; para ello, se tuvo que definir si estas acciones se situaban dentro del modelo rehabilitador o dentro del modelo social de atención a las personas con discapacidad.

El modelo rehabilitador concibe que las personas con discapacidad dependen de los servicios de salud y otros profesionales de referencia, ya que se entiende la discapacidad con causa de algo biológico, por lo tanto, la persona debe ser rehabilitada para participar en la sociedad (Jiménez, 2010, Velarde 2012, Victoria 2013). Es desde este modelo que se crean las carreras de educación especial y terapia física, así como los centros de educación especial, por lo tanto, es un modelo vigente en la actualidad. El camino a seguir en la atención a las personas con discapacidad y sus familias es el modelo social, el cual enfatiza en una perspectiva ecológica, concibe que la discapacidad se genera por la interacción entre las carateristicas de la persona y las respuestas que el medio le ofrece para apoyar su condición (Jiménez, 2010; Palacios, 2008; Velarde 2012, Victoria 2013), por esto, el trabajo de profesionales junto las familias es vital para el desarrollo de las personas con discapacidad.

Este artículo proporciona a quien lo lea: una visión de los modelos de atención a las personas con discapacidad vigentes en nuestro país, el sustento teórico para la conformación de un equipo interdisciplinario basado en el modelo social, fundamentos pedagógicos, propósitos y estrategias de mediación utilizadas en los encuentros del equipo; con la finalidad de construir una comunidad de aprendizaje. Para finalizar reflexionamos sobre los cambios y transformaciones del equipo, así como el devenir que indica el camino a seguir.

El modelo social de atención a las personas con discapacidad está dentro del actual paradigma de derechos humanos, el cual está dirigido a la capacidad de la sociedad para brindar oportunidades de participación. Como lo explica Palacios (2008), las limitaciones dejan de estar en las personas y se transfieren a la sociedad, que se encuentra limitada para prestar servicios y asegurar que las necesidades de la persona con discapacidad sean tomadas en cuenta, se "aboga por la rehabilitación o normalización de una sociedad, pensada y diseñada para hacer frente a las necesidades de todas las personas" (p. 104).

A raíz de los cambios en la concepción de la atención a la población con discapacidad, que se han generado en los últimos años, se inicia una transformación del perfil profesional de fisioterapeutas en el ámbito educativo. Este ha sido respaldado por el Ministerio de Educación Pública, con la publicación de diferentes documentos orientados hacia el modelo social. Estos documentos hacen un llamado claro a realizar la transición del modelo rehabilitador al modelo 
social. Ello implica: partir de las fortalezas del estudiantado; potenciar la participación social; identificar barreras sociales y de infraestructura para disminuirlas o eliminarlas, y construir redes de apoyos alrededor de cada estudiante en los centros o servicios educativos.

Desde estos principios se inició el proceso de transformación, con apoyo en conceptos pedagógicos y en la IAP para encontrar un lugar (mi lugar) profesional en un equipo multidisciplinario de un centro educación especial. El camino es recorrido en compañía de las compañeras de trabajo, que se transforman de participantes a coinvestigadoras. Es también una evolución de un equipo multidisciplinar a interdisciplinar.

La multidisciplina se define como "el estudio de un objeto de una sola y [única] disciplina por varias disciplinas a la vez" (Nicolescu, 1996, p. 37), es el abordaje de la persona por todas las disciplinas, pero sin que exista una comunicación entre profesionales, cada quien trabaja según los objetivos y lo que se enmarca dentro de su especialidad. El trabajo desde una mirada interdisciplinaria se basa en "la transferencia de métodos de una disciplina a otra" (Nicolescu, 1996, p. 37). Esta, en relación con el trabajo colaborativo donde cada participante tiene un papel protagonista, incluidas las familias, es una forma de trabajo acorde con el modelo social, ya que se centra en las personas y sus familias.

Desde una posición basada en el paradigma naturalista, en un equipo de trabajo es necesario tomar en cuenta la existencia de diversas realidades que se interrelacionan, hecho que ayuda a una comprensión más completa del ser humano en su multidimensionalidad (Morin, 2004). Para entender la realidad que vive cada participante del equipo es necesario escuchar lo que tiene que decir, pero con una escucha abierta y sin hacer juicios de valor, se deben reconocer "como personas, con nuestras ideas, sueños, voluntades y pasiones" (Jara, 1994, p. 59).

Con esta premisa se inicia el proceso de investigación. Para Latorre (2004), la investigación en educación debe ser un proceso que lleve a sus profesionales a la autorreflexión, con el objetivo de mejorar su trabajo, es "un proceso reflexivo sobre la propia práctica que lleva a una mayor comprensión de las prácticas y contextos institucionales" (p. 9). La intención de comprender mejor el hacer, condujo a parar, mirar, analizar, reflexionar y actuar, como ciclos recursivos de una espiral.

Al incio el proceso de análisis fue individual, como investigadora llevaba al grupo los datos obtenidos, no era consciente de que le estaba negando al equipo la posibilidad de realizar el análisis de su propias acciones. A pesar de iniciar de esta forma se generó "un diálogo entre saberes: una articulación creadora entre el saber cotidiano y los conocimientos teóricos, que se alimentan mutuamente" (Jara, 1994, p. 40).

Jara (1994) define la sistematización así: 
doi: http://doi.org/10.15359/ree.24-1.20

URL: http://www.una.ac.cr/educare

CORREO: educare@una.cr

Es aquella interpretación crítica de una o varias experiecias que, a partir de su ordenamiento y reconstrucción, descubre o explicita la lógica del proceso vivido, los factores que han intervenido en dicho proceso, cómo se han relacionado entre sí, y por qué lo han hecho de ese modo. (p. 22)

Es decir, la sistematización permite reconstruir las experiencias. Al ordenar los acontecimientos desde la propia cosmovisión y percepción de la realidad, permite relacionar hechos y factores, posibilita el análisis desde la teoría y la práctica, la cual está permeada por la historia de vida de cada de participante. Es así como el equipo interioriza el concepto de sistematización "como una reflexión sobre la experiencia" (Jara, 1994, p. 19).

Para transformar la realidad hay que comprenderla

(Jara, 1994, p. 38)

\section{Metodología}

\section{Fases y estrategias del proceso}

El inicio de la IAP, se da posteriormente a un taller para las familias sobre la temática de la parálisis cerebral. En este taller se brindó un espacio de expresión por medio de dibujos, dinámicas y reflexiones que facilitó que las madres asistentes hablaran de sus sentimientos, aspecto que no se había vivenciado en otros talleres. A raíz de este hecho, las profesionales del equipo iniciaron un proceso reflexivo sobre su práctica y cómo esta repercute en las familias.

Para lograr el intercambio dialéctico, es necesario contar con espacio y tiempo, para planear la actividad a realizar con las familias o evaluar lo sucedido posterior a un taller. La necesidad de estos espacios es evidente, así lo afirma Gallegos (2001): "un lugar donde la gente pueda reunirse solo para hablar, sin tratar de resolver un problema, simplemente para comunicarse y para descubrir si pueden lograr un entendimiento" (p. 102). Se planificaron seis encuentros para realizar la investigación para reflexionar sobre los talleres realizados.

Este tipo de investigación coloca a sus participantes como coinvestigadores o coinvestigadoras, quienes "investigan con y para la gente"(Latorre, 2004, p. 25). Cada participante tiene un papel protagónico al construir la realidad de forma conjunta y recursiva, de esta forma la respuesta a la pregunta inicial surge de la construcción por medio del análisis y la reflexión del equipo.

El equipo interdisciplinario está compuesto por cuatro profesionales que atienden al estudiantado que asiste al grupo de estimulación temprana de discapacidad múltiple. La 
docente de educación especial cuenta con 17 años de experiencia como docente y 9 con población con discapacidad múltiple, tiene una maestría en retos múltiples y sordoceguera, para este artículo la identificaremos como Al. La terapeuta de lenguaje tiene 11 años de experiencia y 6 de trabajar con población con discapacidad múltiple, ha realizado diferentes cursos sobre comunicación con personas con discapacidad, la identificaremos con Mo. Una de las fisioterapeutas es la más joven del grupo tiene 2 años de experiencia, al momento de la investigación cursaba una maestría en estimulación temprana, es su primera experiencia con población con discapacidad múltiple, se identifica como Sa. Por último, queda la investigadora principal, es fisioterapeuta con 14 años de experiencia, siempre ha trabajado con población con discapacidad múltiple y labora en la institución desde hace 4 años, ha realizados diversas formaciones sobre atención fisioterapéutica en pediatría y al momento de la investigación finalizaba la Maestría en Pedagogía con Énfasis en Atención y Desarrollo a la Primera Infancia, la identificaremos como Co.

Este equipo decidió unirse para trabajar, y así atender las necesidades de las familias de estudiantes del nivel de Estimulación Temprana del servicio de Discapacidad Múltiple, no fue por un mandato de parte de la administración del centro educativo. Esto ayudó a la conformación de una comunidad aprendiente, en busca de "construir comunidades humanas sanas sobre principios de competitividad, individualismo, control, interés propio, autoritarismo" (Gallegos, 2001, p. 79).

El proceso investigativo se construyó en dos fases, una diagnóstica y otra de profundización. La fase diagnóstica sirvió para situarse respecto a los intereses de las madres, y permitió iniciar la reflexión sobre la práctica, tomando en cuenta el posicionamiento profesional del equipo frente a las madres. Esta fase requirió de tres encuentros en los cuales se recogieron los datos, se analizaron y se reflexionó sobre la práctica, permitió conocerse desde la perspectiva del trabajo en equipo y comenzar el proceso de aprendizaje, además facilitó situarse frente al proceso de investigación.

La segunda fase fue de profundización, constó de tres encuentros que sirvieron para concretar la participación en el análisis y la reflexión de forma conjunta. Por medio de la sistematización de experiencias se genera un espacio para pensar en la práctica y el resultado ayuda a hacer que las acciones se hagan con conciencia y genera cuestionamientos con mayor profundidad, como el planteamiento de para quién se hacen las cosas, qué se quiere lograr y cómo se hará. El diálogo entre las participantes fue la base del crecimiento, un encuentro entre personas diferente movido por las intenciones sinceras de hacer mejor el trabajo, mediado por el diálogo claro y confiado de las participantes que se saben escuchadas, valoradas y respetadas por su equipo. 
doi: http://doi.org/10.15359/ree.24-1.20

URL: http://www.una.ac.cr/educare

CORREO: educare@una.cr

\section{Estrategias de registro de datos}

Para la sistematización de la experiencia, es necesario utilizar instrumentos adecuados para que la información recolectada brinde los datos para responder a los objetivos de la investigación. Se utilizó la observación participante grabada en audio que"permite la recreación de la interacción verbal y su transcripción" (McKernan, 1999, p. 127), acompañado del diario de campo de la investigadora; también se hizo uso de cuestionarios de preguntas abiertas, que no limitan al sujeto participante y dan libertad de respuesta, así cómo las actividades de análisis grupal con alta implicación de las participantes.

Las estrategias de recolección fueron transformándose en procesos de construcción conjunta, el papel de la investigadora fue proponer actividades para mediar el proceso, con el tiempo el equipo decidió organizar las reuniones a la vez que propuso las estrategias de evaluación donde la participación y la alta implicación de las participantes era más presente. Uno de los aprendizajes más significativos en la primera fase fue pasar de un modelo tradicional de investigación a un modelo participativo enmarcado dentro del paradigma sociocrítico.

Dentro de este paradigma sociocrítico o naturalista cualitativo, es donde esta investigación encuadra de mejor forma, donde la naturaleza de la realidad es compartida por las participantes, es histórica, dinámica y divergente. La finalidad en esta IAP se sitúa dentro de este paradigma, al emancipar sujetos potenciales para el cambio; a la vez que se transforma la realidad, propone una visión de sujeto/objeto íntimamente relacionados, unidos por un fuerte compromiso para el cambio y, al igual que en esta IAP, la investigadora se convierte en una más del equipo (Gurdián-Fernández, 2007).

\section{Diálogo que construye}

Por medio de la sistematización y su respectivo análisis, se extraen hallazgos fundamentales que parten de las afirmaciones de las participantes en cuanto al estado actual del trabajo con las familias y el enfoque que se le da. Esto llevó al equipo a cuestionar, si en realidad sabía lo que hacía, y a la vez a detenerse a observar lo que se estaba haciendo. Al respecto, Jara (1994) enfatiza que la sistematización "permite pensar en lo que se hace, el producto ayuda a hacer las cosas pensadas" (p. 33). De esta forma, se inicia un viaje para conocerse como personas y como equipo, amparadas a la luz de la investigación acción y la sistematización.

El producto de la sistematización se construye a partir de las reflexiones de las participantes, es "compartir críticamente aquellos resultados que surgen de la interpretación de los procesos, de colocar sobre el tapete de la reflexión colectiva los aportes y enseñanzas que se aprenden desde lo que cada quien ha vivido de forma particular" (Jara, 1994, p. 34). 
El aprendizaje se vio reforzado con la metodología utilizada en los encuentros, donde la conversación fue mediada por la investigadora, mediante la presentación de los primeros hallazgos, cuestionarios de preguntas abiertas que fueron discutidos entre todas, y estrategias de validación con alta implicación de las participantes. Además el tiempo cronológico entre los encuentros propició un tiempo personal de reflexión y análisis, facilitando la profundidad y nivel crítico de los aportes durante la propia práctica.

Las categorías de análisis que surgieron del proceso permitieron problematizar las realidades e iniciar la búsqueda colectiva para la reflexión-acción, y fue el constructo para los principales hallazgos que mencionaré a continuación.

\section{Fase diagnóstica: Primer momento}

El primer encuentro fue una conversación larga entre las participantes, sin una guía establecida, su ritmo fue dado por los sentires y pensamientos del equipo. Se dio un proceso fluido, ameno, fue un conversar, para Maturana (1996), "eso se da sin lucha y sin conflicto, solamente con la aceptación del otro" (p. 52). Es por medio de la aceptación del otro ser que podemos escucharnos como un grupo de personas aprendientes, es a partir del propio conocimiento que reflexionamos y podemos iniciar la transformación, una metamorfosis personal que trasciende a lo colectivo como una onda expansiva, no debemos olvidar que "cuando el diálogo se aplica al ámbito educativo, los resultados suelen ser extraordinarios, los valores humanos genuinos despiertan y la espiritualidad brilla (Gallegos, 2001, p. 107).

El proceso de reflexión inicia a raíz de la grabación en audio de la reunión posterior a un taller con las madres de familia, el cual fue transcrito. Se clasificó lo dicho por cada una de las participantes, en temas comunes de los cuales se extrajeron tres grandes categorias: trabajo de las profesionales como parte del equipo de atención en estimulación temprana, roles de las participantes y recursividad que hace referencia a las familias. Esta información fue presentada al equipo para su validación por medio de un análisis a profundidad de cada una de las categorías, el cual se grabó en audio y fue apoyado por las notas de campo de la investigadora.

La necesidad de realizar procesos de sistematización en el trabajo que realiza el equipo se hace evidente en la afirmación de una de las participantes:

\section{Al: Sentimos que estamos haciendo, pero no hay evidencia de las cosas que estamos haciendo.}

Esta frase explica las sensaciones presentes en el equipo, estaban consciente del trabajo con las madres de familia, pero al no dejar evidencia de la labor, se vuelve invisible. No existe material para analizar y reflexionar, se estaba perdiendo la oportunidad de "poder comprender mejor nuestro presente, ubicando - desde la trayectoria acumulada- los elementos, características, contraindicaciones y desafíos de la etapa actual en que nos encontramos" (Jara, 1994, p. 32). 
doi: http://doi.org/10.15359/ree.24-1.20

URL: http://www.una.ac.cr/educare

CORREO: educare@una.cr

Entre los hallazgos del primer análisis se extrae una falta de rumbo, sin objetivos claros, para determinar hacia dónde se quiere caminar, falta de organización para la realización de los talleres, se hace evidente que la elección inicial de los temas se hizo sin tomar en cuenta los intereses reales de las madres de familia, por lo tanto, se detecta un posicionamiento de las profesionales como expertas, enmarcado dentro del modelo rehabilitador y alejado del trabajo colaborativo del modelo social, un ejemplo es la expresión:

Al: En el caso de S es lo trabajada que viene, yo creo que eso es una evaluación para nosotras.

Las profesionales se posicionan como expertas encargadas de dar el conocimiento a las familias y asumen que el comportamiento positivo de una madre frente a la discapacidad de su hija es producto de su trabajo y no de las capacidades de resiliencia de la madre y el apoyo de su entorno.

El equipo toma conciencia de todas las dificultades que tiene tanto de organización como de posicionamiento frente a las familias, es aquí cuando el equipo decide apoyar a la investigadora en su proceso, sin imaginarse que pasarían de ser participantes a coinvestigadoras de su propia labor, así inicia una espiral dialéctica entre la acción y la reflexión.

\section{Segundo momento}

El segundo momento de la fase diagnóstica consistió en conocer las experiencias, los intereses y motivaciones de las participantes para comprender que las movió a unirse a trabajar en equipo para brindar capacitación a las madres de familia. Se realizó por medio de un cuestionario de preguntas abiertas, por que este tipo de "técnicas de investigación permiten recoger datos desde el sistema perceptivo o interpretativo del investigador, solicitar información desde la perspectiva de los participantes y contrastar perspectivas" (Latorre, 2004, p. 55). El propósito se concreta en conocer y poner en común acuerdo lo que nos impulsa a acercarnos a un trabajo interdisciplinar que esté más acorde con el modelo social, por medio de la sistematización de las respuestas y la presentación a las participantes para su análisis y validación.

Entre los hallazgos se encontró la divergencia de criterios sobre si la institución promueve el trabajo en equipo, ya que una de las participantes dice que no se da y otra dice que el trabajo es agradable por el equipo, esto indica que es posible trabajar en equipo, aunque en ocasiones no depende de la institución sino de las personas. Un punto que comparten todas las participantes es la necesidad que muestran por estar en constante formación, generada por incertidumbre de no estar suficientemente preparadas para atender la complejidad de la población y sus familias. 
Se evidenció que el equipo tiene un buen nivel técnico sobre la atención de las necesidades de la población, aspecto que aporta las bases para el intercambio de conocimiento, además muestra actitud de compartir el conocimiento y aprender de la otra persona, de manera que se conforma un equipo multidisciplinar con intenciones y grandes posibilidades de llegar a transformarse en interdisciplinar.

\section{Co: Formación de profesionales da mejor atención}

\section{Tercer momento}

Para la recolección de datos en este encuentro se elaboró de forma conjunta un cuestionario de preguntas abiertas basadas en el análisis de las sistematizaciones anteriores, con el objetivo de reflexionar sobre el trabajo como equipo. La construcción del cuestionario se realizó escuchando las propuestas, mejorandolas y conversando en el equipo sobre lo que se quería saber, luego cada una lo respondió de forma individual.

Del análisis se puede extraer que el equipo tiene capacidad de observar y escuchar, pero de forma individualizada, como característica personal de las participantes y no como un equipo, que lleve a procesos de reflexión frente a lo observado, por lo tanto, las acciones son improductivas e inclusive carentes de sentido, queda pendiente la construcción de una mirada conjunta para mejorar el desempeño.

Se empezaron a abrir espacios de diálogo por medio de la palabra compartida, el ambiente de los encuentros permitió la expresión de sentimientos y temores, se inició la construcción de una comunidad de aprendizaje, que se entiende como el espacio de apoyo, compresión y asesoría por parte de las personas participantes para la "propia indagación interior" (Gallegos, 2001, p. 11). Este crecimiento tanto personal como grupal genera una espiral transformadora de doble vía: cuantos más cambios se den a nivel personal, más cambios a nivel grupal y viceversa.

\section{Fase de profundización: Cuarto momento}

En este encuentro se concretó la participación en el análisis y la reflexión del equipo de forma conjunta. Consistió en hacer un análisis de la sistematización del encuentro anterior. La investigadora agrupó todas las respuestas, pero sin poner el nombre de quien las habia dicho, las presentó al equipo para su análisis y reflexión.

Al preguntarse sobre qué se está haciendo para responder a las necesidades de las madres del servicio de estimulación, todas las participantes brindaron respuestas diferentes, como se evidencia desde el proceso de sistematización. 
doi: http://doi.org/10.15359/ree.24-1.20

URL: http://www.una.ac.cr/educare

CORREO: educare@una.cr

\section{Sa: No hay una sola que coincida}

Es clara la presencia del modelo rehabilitador al preguntar qué es lo que necesitan (nosotras-profesionales) que ellas (madres) reciban. Las profesionales se posicionan como únicas expertas en el desarrollo de la infancia y dejan un rol pasivo a las familias, que se recoge en la siguiente frase.

Mo: Parece que nosotras (profesionales) las vamos a salvar (madres).

Otro de los hallazgos, es que el equipo se encuentra lejos de trabajar en forma colaborativa, por lo tanto, las prácticas realizadas están fuera del modelo social. Esto lleva al equipo a darse cuenta desde dónde se sitúan, pero rescatan la posibilidad de cambiar, es claro por medio de esta frase.

Sa: Es bueno hacer este análisis, porque ahora vamos a cambiar el enfoque.

Al mismo tiempo el equipo se permite pensar sobre aquello que les gustaría encontrarse en su relación profesional. Se percibe un cambio hacia la inclusión de la familia en la comunidad de aprendizaje que se va construyendo, por medio de esta frase.

Al: Quiero ver familias empoderadas, familias resilientes emocionalmente, conocedoras de sus derechos, con estudiantes que maximicen su potencial cognitivo, motor, comunicativo, sensorial.

\section{Quinto momento}

El quinto encuentro se planteó con el objetivo de identificar las estrategias utilizadas por el equipo según los modelos rehabilitador y social. Para lograrlo fue necesaria una estrategia de mediación para definir cada uno de los modelos según el propio conocimiento; cada una escribió en papeles definiciones sobre los modelos, las cuales se clasificaron en grandes categorías y se fueron reduciendo a los aspectos que están relacionados con las familias y con los procesos educativos.

En la información clasificada por las participantes, se observa que no escribieron sobre sus estrategias, sino que la información refleja lo que cada una piensa sobre los modelos. Fue necesario mencionar las características de los modelos para situarse, realizar el análisis y clasificar la información.

En el primer nivel de sistematización la información se pudo clasificar en grupos de temas comunes: capacidades, familia, inclusión, trabajo en equipo, comunicación y aspectos educativos. Mediante el proceso de análisis la información aportada se redujo a dos grandes categorías, el trabajo colaborativo con las familias y los aspectos educativos. 
Cuando se hace referencia a la inclusión de la familia en los programas educativos es necesario que sea el equipo quien abra el espacio para comunicarse con esta y preguntar su opinión respecto a los objetivos, pero sobre todo respetar su decisión.

\section{Sexto momento}

Como parte del proceso de sistematización de experiencias se ve, de forma clara, el rol activo del equipo al utilizar técnicas de sistematización con alta participación en la construcción del conocimiento. Los datos específicos obtenidos fueron el resultado de la conversación sobre las estrategias utilizadas en la práctica enmarcadas según el modelo rehabilitador o social. La dinámica de recolección de la información consistió en que una de las co-investigadoras se ofreció voluntariamente a tomar nota de las afirmaciones. Posteriormente, otra procedió a hablar sobre estrategias utilizadas por el equipo en la atención a la población con discapacidad, así cada una tuvo su espacio para hablar libremente sobre el tema por el tiempo deseado, mientras se tomaba nota de todo. A la vez se fue clasificando las afirmaciones en similares o disimiles.

La dinámica de este ejercicio grupal consistió en revisar los datos escritos y reescribirlos de forma que se puedan agrupar por categorías que sean más completas. Jara (1994) explica claramente que el objetivo de este proceso es la interpretación de forma critica de las experiencias por medio del ordenamiento y la reconstrucción, tomando en cuenta las vivencias, los factores que intervienen y las relaciones entre sí.

Después de pasar por tres niveles de sistematización, se encuentran con temas importantes para realizar la transición del modelo rehabilitador a un modelo social basado en el trabajo colaborativo. Los temas son: Participación activa en el entorno infantil, diálogo respetuoso en el análisis de los objetivos, autoanálisis como generador de transformación, presencia de documentación oficial con enfoque rehabilitador, y superación del egocentrismo profesional.

Dentro del modelo rehabilitador las familias tienen un papel pasivo en los procesos de estudiantes, por esta razón surge la necesidad de trabajar sobre la participación activa en el entorno, lo que se demuestra en esta afirmación:

Mo: Creo que nunca se han sentido parte de los procesos (hace referencia a las familias).

Esta afirmación surge a raíz del cuestionamiento del equipo, en relación con la participación de las familias en los procesos educativos. El equipo se hace consciente de que no le han permitido a las familias tener un papel primordial en la toma de decisiones con respecto a la vida de sus hijos e hijas. Por medio del dialogo respetuoso se brinda la posibilidad de conversar y poner en palabras las emociones, permite mirar desde otro punto nuestras propias acciones. Sobre los hallazgos una de las participantes afirmó: 
doi: http://doi.org/10.15359/ree.24-1.20

URL: http://www.una.ac.cr/educare

CORREO: educare@una.cr

Co: Donde nos pusimos a hablar aquí, vimos que podíamos hacer algo más bonito.

En esta frase se denota cómo el conversar genera otras miradas, y da pie a la transformación desde el analizar, reflexionar, actuar. Para Gallegos (2001), en una comunidad aprendiente el objetivo de todos es aprender.

Otro de los hallazgos fue el análisis respetuoso de los objetivos, esto quiere decir que los objetivos deben ser propuestos respetando los intereses y necesidades de las familias, con las recomendaciones del grupo de especialistas que atienden a la población con discapacidad y colocando al estudiantado y su familia en primer lugar

Es evidente la importancia del trabajo en equipo, de la interdisciplina para una mejor atención a las necesidades de las familias y sobre todo del autoanálisis como generador de transformación. Aquí juega un papel trascendente la transdisciplinariedad definida por Nicolescu, (1996) como lo que está a la vez entre las disciplinas, a través de las disciplinas y más allá de toda disciplina. La docente menciona la importancia de incluir a las terapeutas en las visitas al hogar. Un ejemplo de esto es:

Al: Incluir a las terapeutas en las visitas domiciliarias abre el panorama para brindar recomendaciones que permitan impactar en la vida de los estudiantes. Replantea los objetivos de trabajo.

El equipo, desde un posicionamiento más crítico, reconoce prácticas basadas en el modelo rehabilitador respaldadas por la documentación que regula los mismos servicios. Invita a proponer cambios a corto plazo dentro de lo que puede hacer cada profesional para transitar de modelo en su propia práctica, como hace referencia Mo.

Mo: La normativa de terapia de lenguaje es sumamente rehabilitadora en términos como: rehabilitar, intervenir.

Finalmente, el último supuesto que lleva al análisis es la presencia del egocentrismo profesional, es una huella del modelo rehabilitador en el cual se formó el equipo, es la herencia de prácticas sin cuestionamientos o profundización en su sentido, como lo expresa Al.

Al: "Nos estamos posicionando como magnánimas profesionales que vamos a enseñarles".

Estos resultados nos llevan a volver nuestra mirada a una forma de trabajo que es utilizada en la atención temprana, Ilamada modelo centrado en la familia, del cual podemos tomar sus principios e intentar aplicarlos a la labor dentro de los centros educativos especiales. Este modelo "tiene como objetivo principal capacitar a la familia atendiendo a sus singularidades, resaltando sus fortalezas y confiando en sus elecciones" (Cañadas, 2012, p. 132). 


\section{Conclusiones}

Por la dinámica compleja del trabajo con población de primera infancia con discapacidad múltiple, los equipos interdisiciplinarios deberían abrir espacios de reflexión que permitan, de forma recursiva, analizar la propia práctica. Estos espacios de diálogo que surgen por iniciativa del propio equipo permiten trabajar por un propósito común. Deberían dejarse de lado las identidades profesionales para conocer a la persona, con sus ideas, pensamientos y emociones; el diálogo permite una conexión más cercana que nace desde el amor por la educación y por el ser humano.

El equipo interdisciplinario se va transformando en una comunidad de aprendizaje, donde no existe un líder y todas las personas aprenden de las otras. El reto para la investigadora fue no imponer las ideas propias acerca de lo que pasaba en el equipo y cómo se posicionaban desde el papel de profesionales. La intención fue abrir un espacio de diálogo donde todas las participantes tuvieran la oportunidad de expresar sus sentimientos y pensamientos.

El equipo fue consciente de estar trabajand o dentro del modelo rehabilitador, aunque todas las participantes conocián los principios del modelo social y deseaban trabajar con ese modelo. Se evidenció que las participantes se posicionan como expertas donde elsujeto profesional se siente conocedor de la verdad y puede decirle a la familia lo que tiene que hacer. Proponen la identificación de estas acciones con el fin de poder realizar cambios transformadores y dejar atrás las prácticas del modelo rehabilitador.

Entre la premisas que el equipo debe asumir es necesario aumentar la participación activa en el entorno del infante, esto quiere decir que los cambios deben externderse al entorno próximo de cada persona, su familia y comunidad, no quedarse dentro de las paredes del centro educativo.

Las personas profesionales deben propiciar el diálogo respestuoso con las familias, invitarlas a participar de forma activa en la creación de los objetivos pedagogicos de sus hijos e hijas, los cuales van a incidir en la calidad de vida familiar.

La creación de comunidades de aprendizaje con ejes centrales para el autoanálisis genera tranformación de la propia práctica, estas comunidades deben estar integradas por la familia y los cuerpos profesionales y estar en movimiento continuo de espiral donde cada vez existan más personas involucradas.

El equipo se hace consciente de que dentro de los documentos oficiales es necesario evidenciar vestigios del modelo rehabilitador y solicitar los cambios y, en la medida de lo posible, realizarlos desde la cotidianidad profesional. Las co-investigadoras lo consideran una limitación para el logro de cambios a mayor escala dentro del modelo social. Nace dentro del equipo la inquietud por una propuesta de cambio, basada en prácticas y estrategias amparadas desde un enfoque de derechos, y fundamentadas en la participación social desde el abordaje educativo. 
doi: http://doi.org/10.15359/ree.24-1.20

URL: http://www.una.ac.cr/educare

CORREO: educare@una.cr

Cuando profesionalmente la persona pueden abrirse y valorar el conocimiento presente en el otro ser, es cuando podrán acoger a las familias, dándoles el lugar de protagonistas que tienen en los procesos educativos de sus hijos e hijas, como lo refiere, claramente, el modelo centrado en la familia, donde los grupos profesionales deben aprender a trabajar en colaboración. Son los sujetos profesionales quienes deben ser los primeros en creer en este modelo, confiar en sus acciones para poder transmitirlo a las familias, es dejar de lado el egocentrismo profesional.

Es un reto para el equipo interdisciplinar hacer un cambio drástico en la propia práctica, individual y grupal. Se debe iniciar una onda expansiva de transformación, recordando que cada profesional en los procesos educativos es el de soporte de un proceso vital que cada familia realiza de la mejor forma que puede.

\section{Referencias}

Ávila, A. L., Álvarez, M., Aguilar, C., Sibaja, Z., Villalobos, J. y Esquivel, C. (2005). Normas y procedimientos para el manejo de los servicios educativos para estudiantes con discapacidad múltiple. San José, Costa Rica: Centro Nacional de Recursos para la Inclusión Educativa. Recuperado de https://cenarec.files.wordpress.com/2016/02/normativa-discapacidadmultiple.pdf

Cañadas, M. (2012). La familia, principal protagonista de los centros de desarrollo infantil y atención temprana. Edetania estudios y propuestas socioeducativas, 41, 129-141. Recuperado de https://dialnet.unirioja.es/servlet/articulo?codigo=4089699

Gallegos, R. (2001). La educación del corazón; Doce principios para las escuela holistas. Guadalajara, México: Fundación Internacional para la Educación Holista.

Gurdián-Fernández, A. (2007). El paradigma cualitativo en la investigación socioeducativa. San José, Costa Rica: CECC Y AECl.

Jara, O. (1994). Para sistematizar experiencias: Una propuesta teórica y práctica. San José, Costa Rica: Centro de Estudios y Publicaciones Alforja.

Jiménez, D. P. (2010). Concepto social de la discapacidad. Recuperado de https://www.academia. edu/9652813/CONCEPTO SOCIAL DE LA DISCAPACIDAD

Latorre, A. (2004). La investigación-acción. Conocer y cambiar la práctica educativa (2a ed.). Barcelona: Editorial Grao. 
Maturana, H. (1996). El sentido de lo humano (8a ed.). Santiago: Dolmen ediciones. Recuperado de https://books.google.co.cr/books?id=9Vajw-olPGwC\&pg=PA52\&l $\mathrm{pg}=\mathrm{PA} 52 \& \mathrm{dq}=\mathrm{eso}+\mathrm{se}+\mathrm{da}+\sin +\mathrm{luch} \mathrm{a}+\mathrm{y}+\sin +$ conflicto,+ solamente + con + la + acept aci\%C3\%B3n+del+otro\&source=bl\&ots=gjs6xUm2bl\&sig=ACfU3U1 yiZ6N9gLG1 4SGNLE8pQugUvWfg\&hl=es\&sa=X\&ved=2ahUKEwjms9H94KrjAhWs11 kKHXPFDgQ6AEwB3oECAkQAQ\#v=onepage\&q=eso\%20se $\% 20$ da $\% 20$ sin $\% 20$ lucha $\% 20$ y $\% 20$ sin\%20conflicto\%2C\%20solamente\%20con\%20la\%20aceptaci\%C3\%B3n\%20del\%20 otro\&f=false

McKernan, J. (1999). Investigación-acción y curriculum. Madrid, España: Ediciones Morata.

Morin, E. (2004). La vía. Para el futuro de la humanidad. Barcelona: Paidós.

Nicolescu, B. (1996). La transdiciplinariedad: Manifiesto. México: Multiversalidad Mundo Real Edgar Morin.

Organización Mundial de la Salud (OMS). (2011). Informe mundial sobre la discapacidad (Resumen). Ginebra, Suiza: Autor. Recuperado de https://www.who.int/disabilities/world report/2011/summary es.pdf?ua=1

Palacios, A. (2008). El modelo social de discapacidad: Orígenes, caracterización y plasmación en la Convención Internacional de los Derechos de las Personas con Discapacidad. Madrid: Ediciones Cinca.

Velarde, V. (2012). Los modelos de la discapacidad: Un recorrido historico. Revista Empresa y Humanismo, 15(1), 115-136. Recuperado de https://dadun.unav.edu/ bitstream/10171/29153/1/REYH\%2015-1-12Velarde\%20Lizama.pdf

Victoria, J.A. (2013). Hacia un modelo de atención a la discapacidad basado en los derechos humanos. Boletín Mexicano de Derecho Comparado, 46 (138), 1093-1109. doi: https://doi. org/10.1016/S0041-8633(13)71162-1 